\title{
Phase Dependence on Motion
}

\section{OVERVIEW}

We have seen that the refocusing of spins is equivalent to ensuring that all spins are in phase at the echo. Spin echo sequences have the wonderful property that, even in the presence of local field inhomogeneities, the spins rephase at the Hahn echo time, what we conventionally refer to as $T_{\mathrm{E}}$. The read gradient is then usually designed so that the area under the dephasing portion is exactly balanced by the rephasing portion at the echo time $T_{\mathrm{E}}$. This coincidence of the two echo times, the Hahn echo and the gradient field echo, is usually taken for granted. Ideally then, at least for spin echo sequences, the phase will be zero at each pixel. For the gradient echo (see Fig. B7.1.1), the total phase of spins is zero at the echo time if there is no flow motion in the imaged subject. For the discussions in this unit, we will ignore the effects of field inhomogeneities.

Motion during the gradient moves the spin from one location to another and, therefore, changes the effective field it sees. This field change leads to a frequency change in time during either the dephasing or rephasing gradient, or both. As a result, the spins do not refocus at the expected time. For a simple bipolar pulse (a negative square-pulse gradient of amplitude $G$ followed immediately by a positive square-pulse gradient of amplitude $G$, see Fig. B7.1.1) and spins moving with a constant speed, the change in phase $(\phi)$ for motion through the gradients is given in terms of $\gamma$, the gyromagnetic ratio, $G$, the above mentioned gradient amplitude, the gradient pulse time $\tau$ for each lobe, and the speed of the spins during this time, $v$ :

$$
\phi=-\gamma G v \tau^{2}
$$

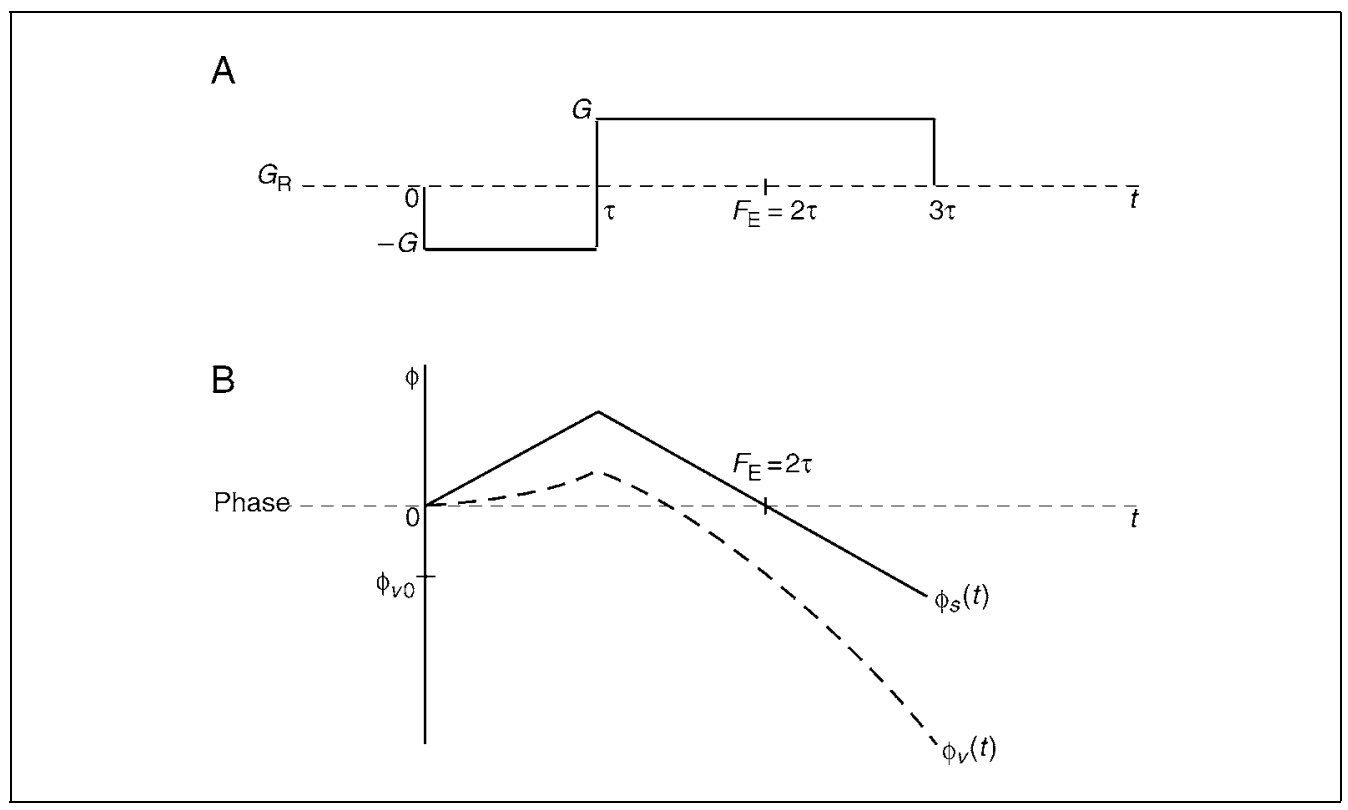

Figure B7.1.1 (A) A typical bipolar, square-pulse readout gradient waveform whose zeroth moment vanishes at the field echo time $F_{\mathrm{E}}$. Recall that the negative lobe is referred to as the dephasing lobe of the read gradient and the positive lobe as the rephasing lobe. (B) The phase accumulated as a function of time for stationary spins (solid line) and the additional phase for spins moving with a constant velocity (dashed line) along the direction of the gradient $G$. The actual value of the phase at the echo for the constant velocity case depends on the speed, gradient and gradient timings. The symbol $F_{\mathrm{E}}$ refers to the field echo time, that is, the time from the beginning of the dephasing lobe to the gradient field echo.

Contributed by Yu-Chung Norman Cheng and E. Mark Haacke

Current Protocols in Magnetic Resonance Imaging (2005) B7.1.1-B7.1.13

Copyright $(\mathcal{C} 2005$ by John Wiley \& Sons, Inc.
Motion Artifacts,

Motion

Compensation and Magnetic

Resonance

Angiography

\section{B7.1.1}

Supplement 13 


\section{A}

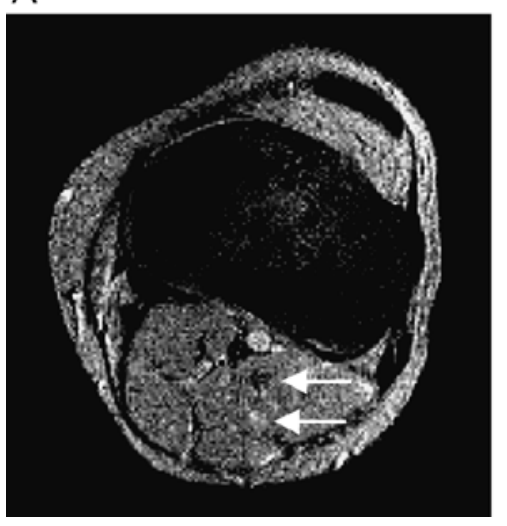

\section{B}

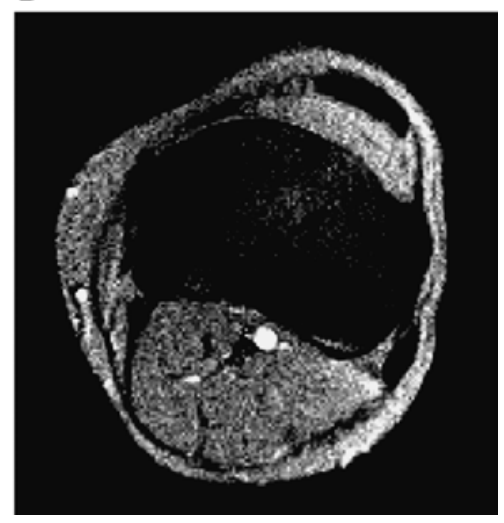

Figure B7.1.2 Velocity compensation in the slice select direction. Transverse image of the knee obtained with (A) an uncompensated slice select gradient structure and (B) a compensated slice select gradient structure. The uncompensated image acquisition shows ghosting (arrows) as well as some dephasing and consequent signal loss whereas both these effects are removed by velocity compensation. The cancellation of signal from the ghosts in some cases (rather than coherent addition to the background) occurs because the phase of that particular ghost is negative (relative to the background). Imaging parameters: (A) $T_{\mathrm{R}} / T_{\mathrm{E}}=15 \mathrm{msec} / 5 \mathrm{msec}, T_{s}=2.56 \mathrm{msec}, \Delta \mathrm{x} \times \Delta \mathrm{y} \times$ $\Delta \mathrm{z}=0.78 \mathrm{~mm} \times 0.78 \mathrm{~mm} \times 5.0 \mathrm{~mm}, N_{\mathrm{x}} \times N_{\mathrm{y}}=256 \times 256, \theta=5^{\circ}, N_{\mathrm{acq}}=1, \tau_{\mathrm{RF}}=1.024 \mathrm{msec}$, $G_{s s}=9.6 \mathrm{mT} / \mathrm{m}$; (B) the same as in (A) except that $T_{\mathrm{R}} / T_{\mathrm{E}}=14 \mathrm{msec} / 4.8 \mathrm{msec}, \tau_{\mathrm{RF}}=1.0 \mathrm{msec}$, $G_{s s}=7.2 \mathrm{mT} / \mathrm{m}$.

\section{A}

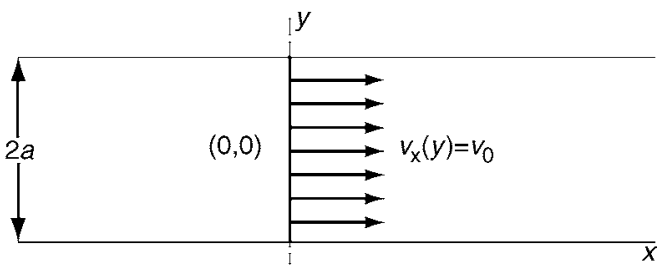

$\mathrm{B}$

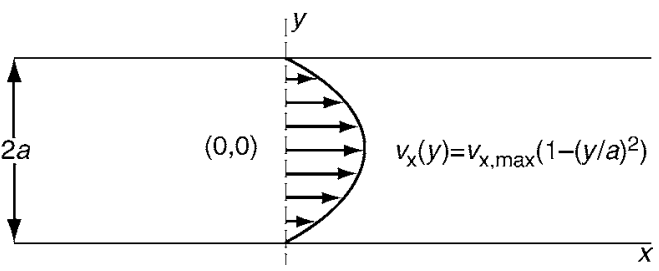

Figure B7.1.3 Illustration of a (A) plug-flow velocity profile and (B) laminar-flow velocity profile for a cross-sectional cut through a cylindrical vessel.

The presence of phase by itself is not a bad thing. Phase can be used to measure flow under the right circumstances (see Equation B7.1.1) or local magnetic field, for example. The problem arises when the velocity, and hence phase, changes in time, or if there is a significant phase change across the voxel because of velocity dispersion across the voxel. The first will cause ghosting, while the second will cause signal loss from intravoxel dephasing. The pulsatility of the blood flow during the cardiac cycle causes changes in velocity as a function of time and is a source of the first type of artifact. Figure B7.1.2

Phase Dependence on Motion

B7.1.2 shows an example of ghosting artifacts in the leg of a volunteer. Laminar flow (see Fig. B7.1.3) is an example of spatial changes in velocity and leads to the second type of artifact. 


\section{A}

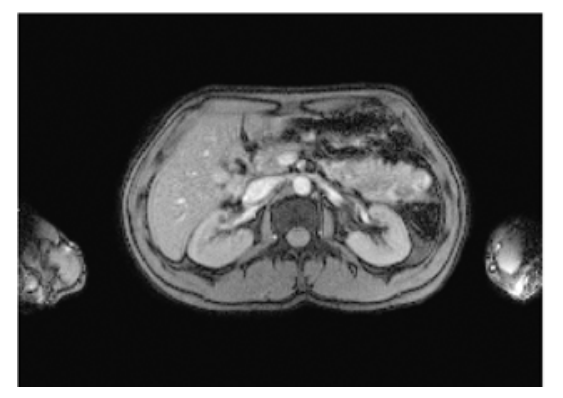

B

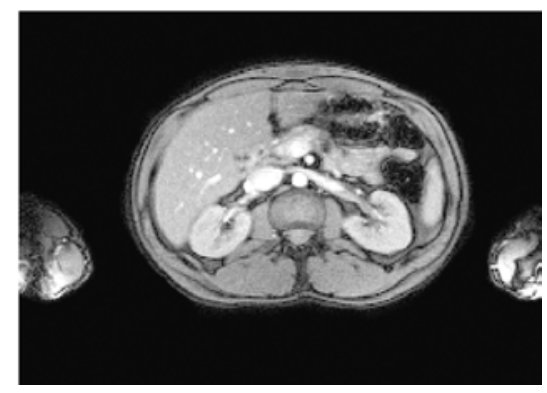

Figure B7.1.4 Ghosting artifacts due to periodic translational motion such as respiration can be eliminated either by acquiring the entire data set over a short period of time or by avoiding the motion. (A) When a $T_{\mathrm{R}}$ of $50 \mathrm{msec}$ is used, the acquisition time is on the order of only three breaths, and the ghosting artifact is imperceptible. Instead, it is replaced by a small blur of the edge features where motion occurs. (B) With a breath-hold, the chest wall motion is completely avoided and negligible blurring occurs. Using the same $T_{\mathrm{R}}$ of $50 \mathrm{msec}$, the entire slice can be acquired in a short breath-hold period (in this case, $\sim 13 \mathrm{sec}$ ). Now, there are no ghosting artifacts or blurring, although subject cooperation is required. Imaging parameters: (A), (B) $T_{\mathrm{R}} / T_{\mathrm{E}}=50 \mathrm{msec} / 12 \mathrm{msec}$, $T_{s}=7.68 \mathrm{msec}, \Delta x \times \Delta y \times \Delta z=1.95 \times 1.95 \times 10.0 \mathrm{~mm}, N_{\mathrm{x}} \times N_{\mathrm{y}}=256 \times 256, \theta=45^{\circ}, N_{\mathrm{acq}}$ $=1, \tau_{\mathrm{RF}}=2.56 \mathrm{msec}, G_{s s}=2.4 \mathrm{mT} / \mathrm{m}$.

The sources of these phase changes can come from the read gradient, from the slice select gradient, and from the phase encoding gradient. For respiratory or cardiac motion, the timing of the RF pulses is not guaranteed to be in synchronization with the period of these two motions unless respiratory gating or cardiac triggering is used to do so. Examples of these periodic motion effects are shown in Figures B7.1.4, B7.1.5, and B7.1.6. If these last two procedures are used, the acquisition times often increase significantly. (Although there is no avoiding this if the goal is to image the heart itself, it is not necessary for, say, imaging the body or most other body parts.) The use of short $T_{\mathrm{R}}$ scans or other fast imaging methods are being used more often today, and they will be susceptible to phase changes in time when these timing mechanisms are not feasible. Generally, ghosting and signal loss will then result.

There are also two other sources of error in the images caused by flow. One is caused by the motion of the spins between the phase (or partition) encoding and the echo, and is referred to as a misregistration artifact. This implies that the vessel appears shifted in the image from its actual location spatially. This phenomenon is demonstrated in the schematic drawing in Figure B7.1.7B, for a phantom in Figure B7.1.8A, and in a neck example in Figure B7.1.9. The shift in position of spins leads to a bright line artifact shown in the last two figures. The second comes about from the motion during the read gradient itself. Even if motion at the echo is compensated, this effect remains and can cause a blurring of the vessel.

All artifacts but the latter can be eliminated for constant velocity motion or flow if the phase at the echo can be made constant independent of velocity. Evidently, by adding another gradient lobe to the usual gradient structure, the effects of constant velocity flow can be removed and the phase once again refocused as if the spins were not moving. (This topic is discussed in UNIT 7.2.)

Motion Artifacts, Motion Compensation and Magnetic Resonance Angiography

\section{B7.1.3}

Supplement 13 


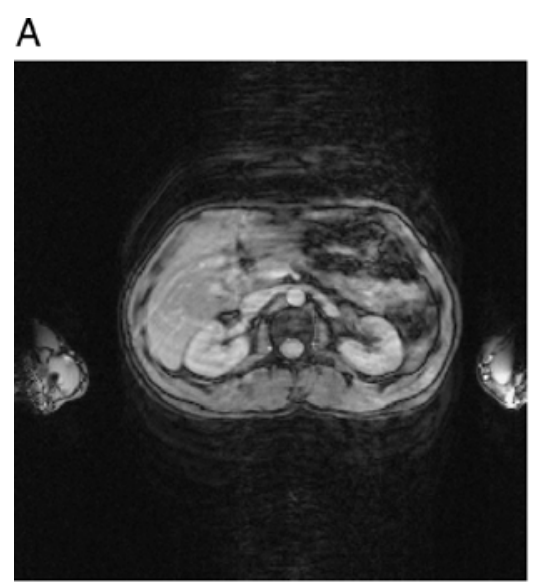

C

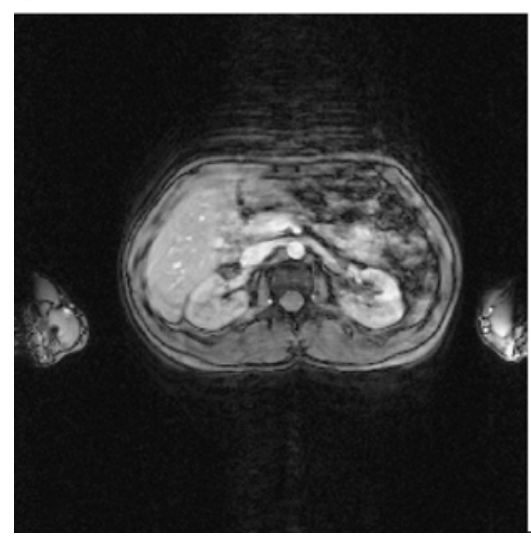

B

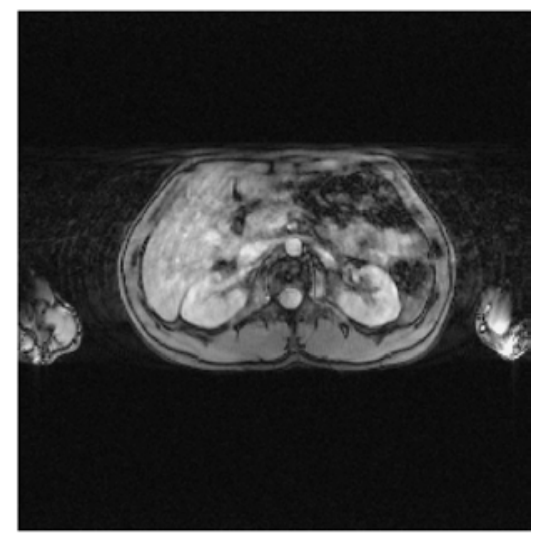

D

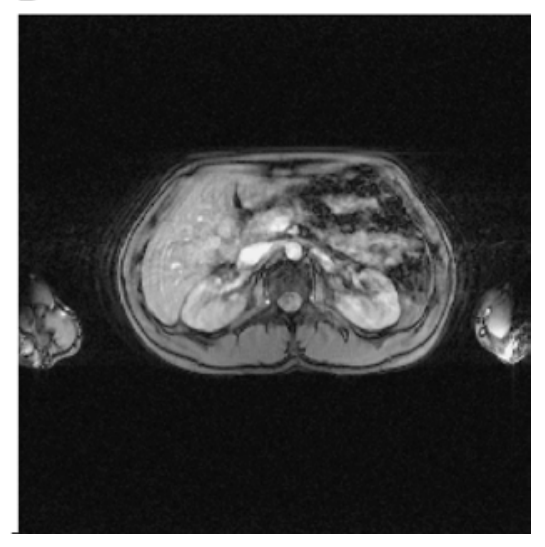

Figure B7.1.5 Simple periodic translational motion, such as that in respiration, leads to ghosting. A gradient echo sequence compensated in both read and slice select directions still exhibits ghosting. Images (A) and (B) were collected with a $T_{R}$ of $400 \mathrm{msec}$ while images (C) and (D) were collected with a $T_{R}$ of $200 \mathrm{msec}$. Images $(A)$ and $(C)$ were acquired with the phase encoding direction in the vertical direction along which chest wall motion occurs during respiration. Images (B) and (D) are acquisitions of the same 2-D slice with the read direction now along the direction of motion. In both cases, the ghosts appear along the phase encoding direction and their positions are determined according to Equation B7.1.25. Imaging parameters: $(A),(B) T_{R}=400 \mathrm{msec}$, $T_{s}=7.68 \mathrm{msec}, \Delta x \times \Delta y \times \Delta z=1.95 \times 1.95 \times 10.0 \mathrm{~mm}, N_{\mathrm{x}} \times N_{\mathrm{y}}=256 \times 256$, $\theta=45^{\circ}, N_{\mathrm{acq}}=1, \tau_{\mathrm{RF}}=2.56 \mathrm{msec}, G_{s s}=2.4 \mathrm{mT} / \mathrm{m}$; (C), (D) the same as in (A), (B) except that $T_{\mathrm{R}} / T_{\mathrm{E}}=200 \mathrm{msec} / 12 \mathrm{msec}$.

Phase Dependence on Motion

\section{B7.1.4}

\section{TECHNICAL DISCUSSION}

Spin Phase Caused by Constant Velocity Flow or Motion in the Read Direction for a Bipolar Square Pulse Example

We start by examining the phase behavior of the spins during the dephasing and rephasing lobes of the read gradient. The easiest motion to evaluate is simple translational motion of an isochromat of spins during sampling:

$$
x(t)=x_{0}+v_{x} t
$$

where $x_{0}$ is the initial position at time $t=0$ and $v_{x}$ is the uniform velocity within the isochromat. These spins see a changing field as they move through the gradient. The 

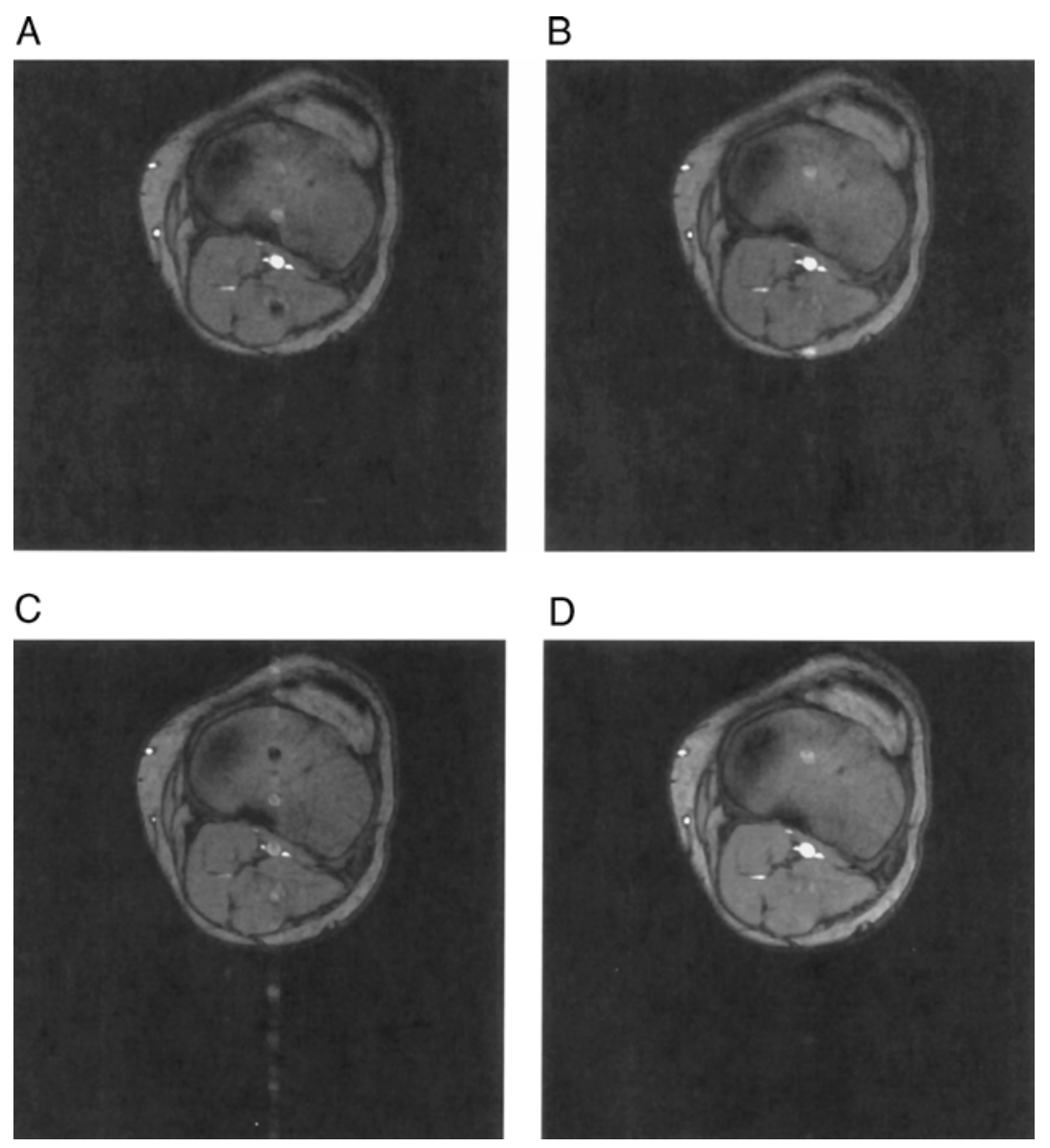

Figure B7.1.6 Transverse images of the knee showing the $T_{\mathrm{R}}$ dependence of the ghosting of the vessel signal in the phase encoding direction due to through-plane flow. (A) $T_{\mathrm{R}}=14 \mathrm{msec}, N_{\text {acq }}=$ 1, (B) $T_{\mathrm{R}}=14 \mathrm{msec}, N_{\mathrm{acq}}=2$, (C) $T_{\mathrm{R}}=14 \mathrm{msec}, N_{\mathrm{acq}}=1$, with no velocity compensation (all other images shown here are velocity compensated), and (D) $T_{\mathrm{R}}=28 \mathrm{msec}, N_{\mathrm{acq}}=1$. Generally, as $T_{R}$ (or the effective $T_{R}$ ) increases the spacing between the ghosts increases-compare (B) and (D) to (A). Imaging parameters: (A) $T_{\mathrm{R}} / T_{\mathrm{E}}=14 \mathrm{msec} / 7.0 \mathrm{msec}, T_{s}=2.56 \mathrm{msec}, \Delta \mathrm{x} \times \Delta \mathrm{y} \times \Delta \mathrm{z}=$ $0.78 \times 0.78 \times 5.0 \mathrm{~mm}, N_{\mathrm{x}} \times N_{\mathrm{y}}=512 \times 512, \theta=30^{\circ}, N_{\mathrm{acq}}=1, \tau_{\mathrm{RF}}=1.0 \mathrm{msec}, G_{s s}=7.2 \mathrm{mT} / \mathrm{m}$; (B) $N_{\mathrm{acq}}=2$; (C) $\tau_{\mathrm{RF}}=1.024 \mathrm{msec}, G_{s s}=9.6 \mathrm{mT} / \mathrm{m}$; (D) $T_{\mathrm{R}}=28 \mathrm{msec}, N_{\mathrm{acq}}=1$.

phase behavior during the dephasing lobe of the read gradient (see Fig. B7.1.1A) is given by:

$$
\begin{aligned}
\phi_{-}(t) & =-\int d t \omega(t)=-\gamma \int d t B(t) \\
& =-\gamma \int_{0}^{t} d t^{\prime \prime} G_{x}\left(t^{\prime \prime}\right) x\left(t^{\prime \prime}\right) \quad 0 \leq t \leq \tau \\
& =\gamma G\left(x_{0} t+\frac{1}{2} v_{x} t^{2}\right)
\end{aligned}
$$

Hence, at the end of the dephasing lobe, the phase is:

$$
\phi_{-}(\tau)=\gamma G x_{0} \tau+\frac{1}{2} \gamma G v_{x} \tau^{2}
$$

Motion Artifacts, Motion

Compensation and Magnetic

Resonance Angiography

\section{B7.1.5}

Supplement 13 


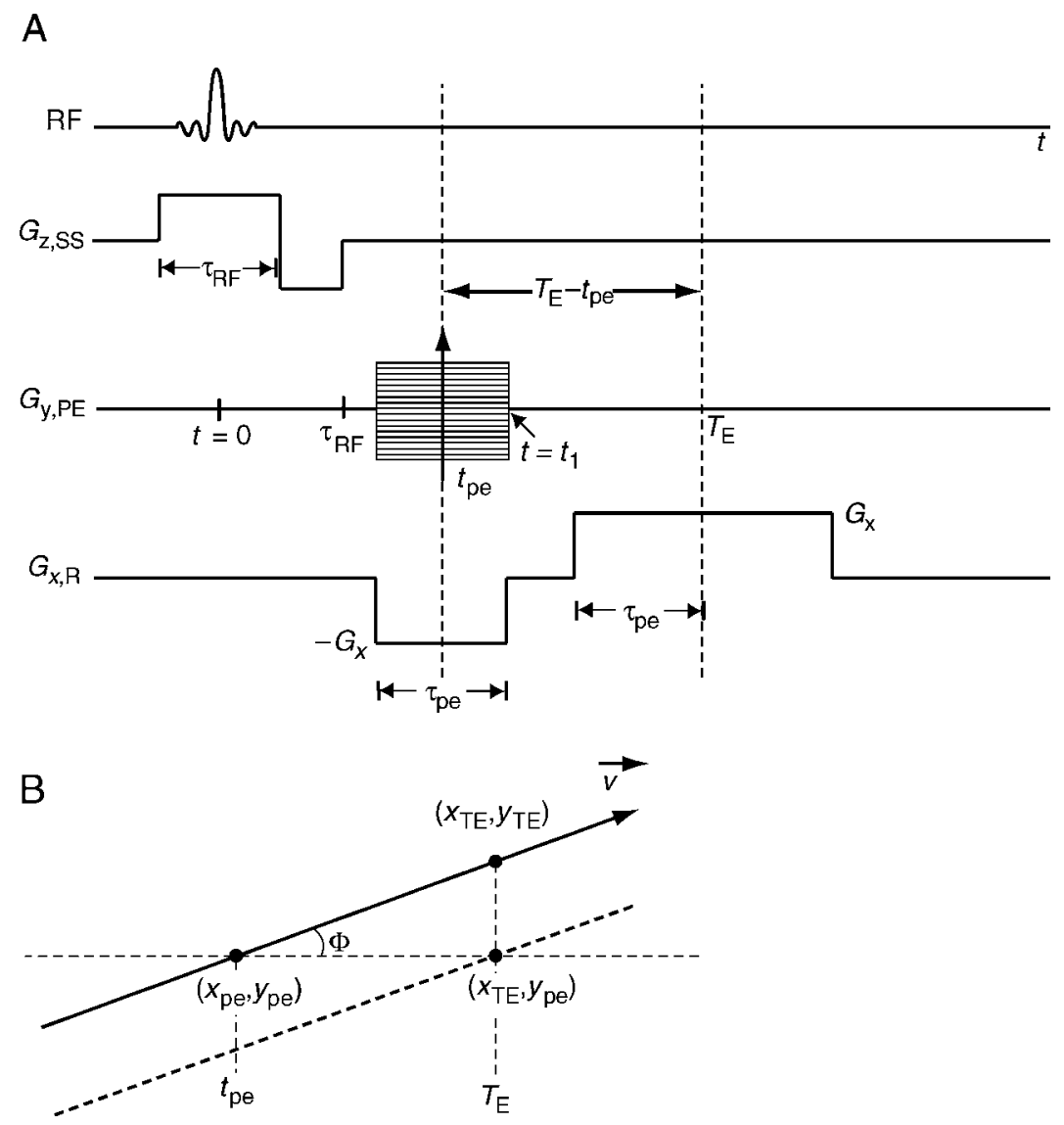

Figure B7.1.7 Illustration of $(\mathbf{A})$ the time line of the gradient structures, including the time difference $\left(T_{\mathrm{E}}-t_{\mathrm{pe}}\right)$ between the effective time when phase encoding occurs and the echo time, and (B) the misregistration artifact due to oblique flow. The solid line represents the physical path of the spins. The dashed line is where the same spins appear in the MR image. Spins at the physical location $\left(x_{\mathrm{TE}}, y_{\mathrm{TE}}\right)$ appear at their encoded position $\left(x_{\mathrm{TE}}, y_{\mathrm{pe}}\right)$ in the resulting image.

The additional phase accumulated from $\tau$ to a time $t$ during the rephasing of the read gradient is:

$$
\begin{aligned}
\phi_{+}(t) & =-\gamma \int_{\tau}^{t} d t^{\prime \prime} G_{x}\left(t^{\prime \prime}\right) x\left(t^{\prime \prime}\right) \\
& =-\gamma G x_{0}(t-\tau)-\frac{1}{2} \gamma G v_{x}\left(t^{2}-\tau^{2}\right) \quad \tau \leq t \leq 3 \tau
\end{aligned}
$$

and the total phase accumulated is given by:

$$
\begin{aligned}
\phi(t) & =\phi_{-}(\tau)+\phi_{+}(\tau) \\
& =-\gamma G x_{0}(t-2 \tau)-\frac{1}{2} \gamma G v_{x}\left(t^{2}-2 \tau^{2}\right) \quad \tau \leq t \leq 3 \tau
\end{aligned}
$$

Using $t^{\prime}=t-2 \tau$ (i.e., shifting the time origin to the expected echo time $2 \tau$ ), leads to:

$$
\begin{aligned}
\phi\left(t^{\prime}\right) & =-\gamma G x_{0} t^{\prime}-\frac{1}{2} \gamma G v_{x}\left(t^{2}+4 \tau t^{\prime}+2 \tau^{2}\right) \quad-\tau \leq t^{\prime} \leq \tau \\
& =-\gamma G\left(x_{0}+2 v_{x} \tau\right) t^{\prime}-\gamma G \nu \tau_{x}^{2}-\frac{1}{2} \gamma G v_{x} t^{\prime}
\end{aligned}
$$

B7.1.6 
A

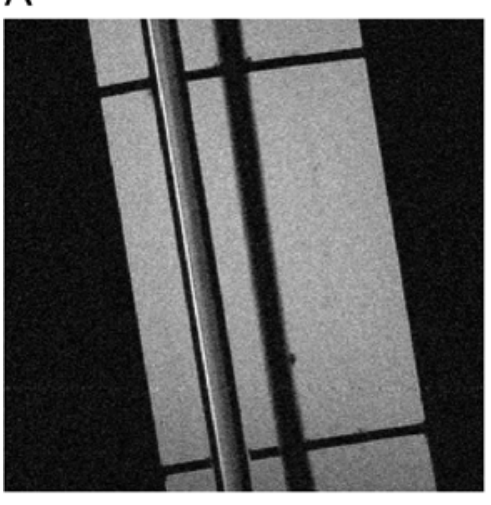

C

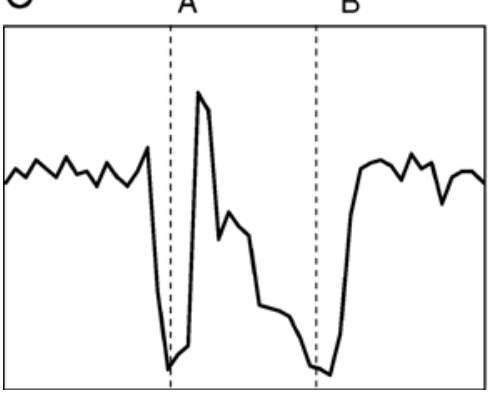

B
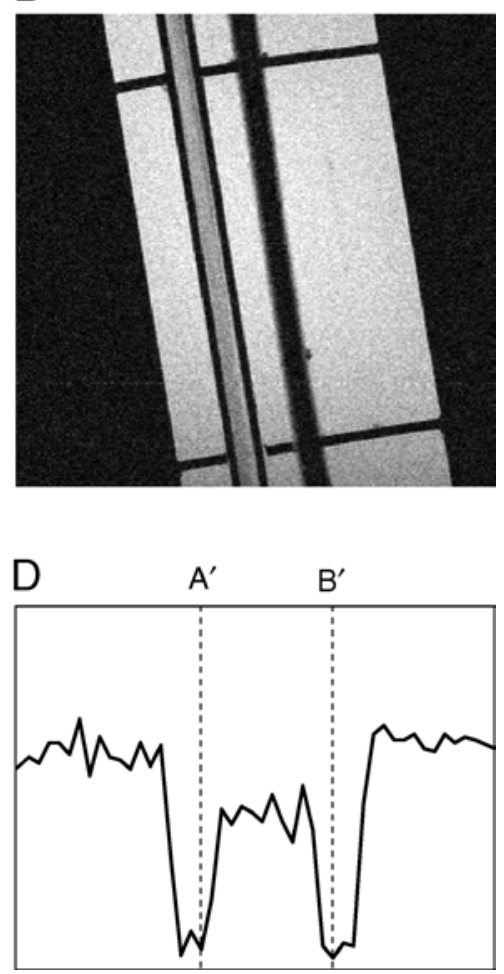

Figure B7.1.8 Images showing an oblique cylindrical tube containing flowing material. The flow is upward along the axis of the tube-i.e., in the plane of the image. The images were obtained with a sequence that has no velocity compensating structure in the phase encoding direction. Images obtained with a short $T_{\mathrm{R}}$ 2-D gradient echo sequence which is uncompensated in the phase encoding direction (image A), and with a fully compensated sequence (image B). The profiles across the flowing material for these two images are shown in (C) and (D), respectively. The boundaries between the flowing material and the surrounding no-signal region are demarcated as $A, B, A^{\prime}$, and $B^{\prime}$ in graphs (C) and (D). The large peak in the profile in (C) represents spin signal shifted to the left. The flat profile between $A^{\prime}$ and $B^{\prime}$ in (D) indicates a successful velocity compensation along the phase encoding (horizontal) direction. Imaging parameters: (A), (B) $T_{\mathrm{R}} / T_{\mathrm{E}}=$ $60 \mathrm{msec} / 28 \mathrm{msec}, T_{s}=12.8 \mathrm{msec}, \Delta x \times \Delta y \times \Delta z=0.59 \times 0.59 \times 2.0 \mathrm{~mm}, N_{\mathrm{x}} \times N_{\mathrm{y}}=512 \times$ 256, $\theta=25^{\circ}, N_{\mathrm{acq}}=4, \tau_{\mathrm{RF}}=5.12 \mathrm{msec}, G_{\mathrm{ss}}=9.0 \mathrm{mT} / \mathrm{m}$.

Rewriting Equation B7.1.7 in terms of its stationary, $s$, and velocity, $v$, components gives:

$$
\phi\left(t^{\prime}\right)=\phi_{s}\left(t^{\prime}\right)+\phi_{v}\left(t^{\prime}\right)
$$

where:

$$
\phi_{s}\left(t^{\prime}\right)=-\gamma G x_{0} t^{\prime}
$$

and:

$$
\phi_{\nu}\left(t^{\prime}\right)=-\frac{1}{2} \gamma G v_{x}\left(2 \tau^{2}+4 \tau t^{\prime}+t^{\prime 2}\right)
$$

The phase behavior for $\phi_{s}\left(t^{\prime}\right)$ and $\phi_{v}\left(t^{\prime}\right)$ is plotted in Figure B7.1.1B, demonstrating that the phase of a stationary spin is zero at the echo, but the phase for a moving spin does not refocus at $t^{\prime}=0$ for a conventional gradient echo. Specifically, at the echo in the

Motion Artifacts, Motion

Compensation and Magnetic

Resonance Angiography

\section{B7.1.7}

Supplement 13 
$t^{\prime}$ frame (i.e., the first term in Equation B7.1.10), the phase for a constant velocity spin is:

$$
\phi_{v 0} \equiv \phi_{v}(0)=-\gamma G v_{x} \tau^{2}
$$

The middle term, $-\gamma G v_{x} 2 \tau t^{\prime}$, looks suspiciously similar to $\phi_{s}\left(t^{\prime}\right)$. Adding these two together yields the phase:

$$
\tilde{\phi}\left(t^{\prime}\right)=-\gamma G t^{\prime}\left(x_{0}+2 \tau v_{x}\right)
$$

or

$$
\tilde{\phi}\left(k_{x}\right)=-2 \pi k_{x}\left(x_{0}+2 \tau v_{x}\right)
$$

which basically determines the position at which the spin is recorded. The term $2 \tau v_{x}$ can be easily understood as a position shift equal to the distance the spin moves from the beginning of the readout procedure to the echo. Finally, the term $-\gamma v_{x} t^{2}$ acts as a filter on the signal which leads to blurring and some potential signal loss.

It is worth reviewing some numerical examples. The phase effects will depend not only on $v_{x}$ but also on the imaging parameters $\tau$ and $\Delta x$. This can be seen by rewriting Equation B7.1.11 using the Nyquist condition:

$$
¥ G \Delta x T_{s}=\ngtr G \Delta x(2 \tau)=1
$$

to give:

$$
\phi_{v 0}=-\frac{\pi v_{x} \tau}{\Delta x}
$$

For $\tau=2.5 \mathrm{msec}$ and $\Delta x=1 \mathrm{~mm}$, the phase will be $-2 \pi$ for a speed of $80 \mathrm{~cm} / \mathrm{sec}$. Even a flow of just $10 \mathrm{~cm} / \mathrm{sec}$ will produce a phase of $\pi / 4$. For an SNR (signal-to-noise ratio) of $10: 1$, the phase error is 0.1 radian and $\pi / 4$ is easily measured.

If the read gradient is the only gradient on, as is usually the case during data sampling, then only the $x$ component of the speed, $v_{x}$, plays a role in causing a phase shift. That is, only the projection $\vec{v} \cdot \vec{G}_{\text {read }}=v_{x} \mathrm{G}_{\text {read }}$ causes the effects described. The effect of spins moving along other axes will be discussed later.

For the bipolar pulse in Figure B7.1.1A, the phase produced by a flow with a constant acceleration, $a_{x}$, at the field echo time $F_{\mathrm{E}}\left(t^{\prime}=0\right)$ can be calculated by similar mathematical steps from Equation B7.1.3 to Equation B7.1.11. If the motion of spins is assumed to be described by:

$$
x(t)=x_{0}+v_{x} t+\frac{1}{2} a_{x} t^{2}
$$

then the term associated with acceleration $a_{x}$ of the phase at the echo is $-\gamma G a_{x} \tau^{3}$. A similar term, also dependent on time cubed, exists even if the sequence is velocity compensated.

\section{Spin Phase Caused by Flow or Motion in the Slice Select Direction}

Phase Dependence on Motion B7.1.8

The slice select gradient also naturally looks like a bipolar gradient design (see Fig. B7.1.7). By assuming that spins are effectively tilted into the transverse plane at the center of the RF pulse, the spins will dephase during the time $\tau_{\mathrm{RF}} / 2$ during which the 
slice select gradient is still on. Stationary spins refocus during the rephasing lobe during the next $\tau_{\mathrm{RF}} / 2$ time interval. Again, the phase behaves according to Equation B7.1.11 but with $\tau$ replaced by $\tau_{\mathrm{RF}} / 2$.

\section{Spin Phase Caused by Flow or Motion in the Phase Encoding Direction}

The phase behavior along the phase encoding direction is similar to the phase accumulation along the read direction discussed above. If a flow is along the phase encoding direction, $y$, then we can replace the variable $x$ by the variable $y$ in Equation B7.1.2 and also replace $v_{x}$ by $v_{y}$, where $v_{y}$ is a constant velocity of the flow in the phase encoding direction. The phase accumulation in the phase encoding direction is then similar to the formula given in Equation B7.1.3, with the read gradient $G_{x}$ replaced by the phase encoding gradient $G_{y}$. Usually, $G_{y}$ is a constant through each phase encoding step and this makes the calculation of phase accumulation in the phase encoding direction simpler than the calculation of phase in the read direction; however, one should pay attention to the fact that the phase encoding table in a sequence occurs at a finite time before the echo (see Fig. B7.1.7). To understand the effects of motion along $\hat{y}$, these timings must be considered.

\section{Effects of constant velocity flow in the phase encoding direction: The misregistration artifact}

The main effect of flow parallel to the phase encoding direction is one of spatial misregistration. Spins along each axis in the reconstructed image appear at the location where they were at the time they were encoded by the associated gradient. If they continue to move before the data are collected, they will still appear at the position where they were encoded. For example, consider phase encoding a moving spin at a time $t_{\mathrm{pe}}$ (assume phase encoding to be instantaneous) when the spin's $y$ position is $y_{\text {pe }}$. Some time later, at the echo, the data for the same spin are collected, but the spin has moved to a new position $y_{\mathrm{TE}}$. In the reconstructed image, the spin will appear at the $y$ position, $y_{\mathrm{pe}}$, where it was encoded. This leads to a spatial misregistration artifact in the resulting reconstructed image. A common manifestation of this artifact occurs for an in-plane vessel, with velocity components along the $x$ and $y$ axes as shown in Figure B7.1.7.

In Figure B7.1.7B, the physical location of a particular spin is plotted at different times. It is seen that the spin is phase encoded at the position $y_{\mathrm{pe}}$, at a time $t_{\mathrm{pe}}$. Its $x$ position is encoded later at the echo, when data are collected at the position $x_{\mathrm{T}_{\mathrm{E}}}$. Therefore, in the resulting image, the spin appears at the point $\left(x_{\mathrm{T}_{\mathrm{E}}}, y_{\mathrm{pe}}\right)$, at the location where information for each axis was encoded, but a location that the spin never physically occupies.

Usually, it is assumed that the spin should appear in the image at the physical location it occupies at the echo, but the spin is shifted from its physical location in the $y$ direction because it moves between the time it is phase encoded and the time the data are read. Given a spin with a constant $y$ velocity, the shift of the spin along $y$ in the image can be calculated from:

$$
\Delta y_{v}=-v_{y}\left(T_{\mathrm{E}}-t_{\mathrm{pe}}\right)
$$

The direction of the shift is dependent on the direction of flow but independent of the point along a vessel (see Fig. B7.1.9). For plug flow, the whole vessel is shifted or misregistered in the image. In Figure B7.1.9A, the read gradient is horizontal and flow in the phase encoding direction leads to misregistration in that direction (see the blurring of the signal in the vertical direction, i.e., the bright bands). In Figure B7.1.9B, the phase encoding is horizontal and the bright bands now appear on the left/right sides of the vessel. If flow is in the reverse direction to that shown in Figure B7.1.7, the shift is up instead. For $T_{\mathrm{E}}$

Motion Artifacts, Motion Compensation and Magnetic Resonance Angiography

B7.1.9 

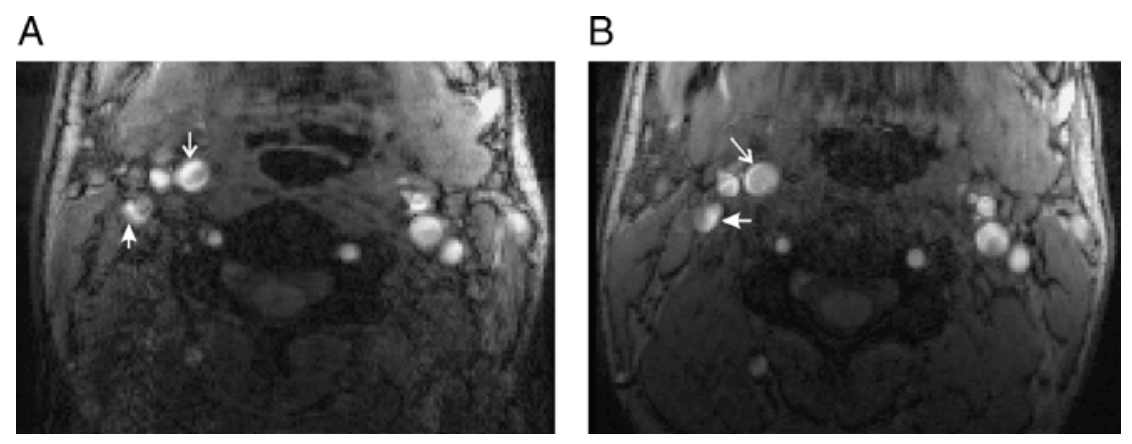

Figure B7.1.9 Images showing an oblique vessel imaged with a normal (single-lobe) phase encoding gradient structure. In (A), the read direction is in the left-right direction while in $(\mathbf{B})$ it is in the up-down direction. Note the misregistration artifact (as shown by the arrows) changes its direction as the phase encoding direction changes. Imaging parameters: (A), (B) $T_{\mathrm{R}} / T_{\mathrm{E}}=27$ $\mathrm{msec} / 11.25 \mathrm{msec}, T_{s}=10.24 \mathrm{msec}, \Delta x \times \Delta y \times \Delta z=0.78 \times 0.78 \times 1.0 \mathrm{~mm}, N_{\mathrm{x}} \times N_{\mathrm{y}} \times$ $N_{\mathrm{z}}=512 \times 384 \times 50, \theta=20^{\circ}, N_{\mathrm{acq}}=1, \tau_{\mathrm{RF}}=1.28 \mathrm{t} \mathrm{msec}, G_{s s}=4.4 \mathrm{mT} / \mathrm{m}$.

$-t_{\mathrm{pe}}=5 \mathrm{msec}$, and a flow of $80 \mathrm{~cm} / \mathrm{sec}$, the misregistration is $4 \mathrm{~mm}$ (up to a four pixel shift). When a vessel shifts this far, it leaves behind zero signal (i.e., there is a black line where the vessel used to be) and it enhances the signal for the tissue on which it now sits. Methods of flow compensation to reverse this artifact are discussed in UNIT B7.2.

\section{Phase variation view of the shift artifact and the effective time of phase encoding}

A signal processing view of the misregistration problem adds further insight into this vessel-shifting effect. Recall from the Fourier transform shift theorem that such a constant shift occurs in the image if the $k$-space data has a linear phase shift proportional to the shift in the image domain. The correct $y$ position of the isochromat at $t=T_{\mathrm{E}}$ (the time of spatial encoding in the $x$ direction and data measurement) was assumed to be:

$$
y\left(T_{\mathrm{E}}\right)=y_{\mathrm{pe}}+v_{y}\left(T_{\mathrm{E}}-t_{\mathrm{pe}}\right)
$$

This implies that to find the shift of the vessel coordinates relative to this actual position, the extra phase accumulated by moving spins must be determined relative to a time origin $t^{\prime}=0$, such that $t^{\prime}=t-T_{\mathrm{E}}$. Time origin matters for the phase encoding gradient first moment calculation because it has a nonzero zeroth moment (see UNIT B7.2). The phase accumulated by constant velocity spins needs to be calculated during a phase encoding gradient waveform on a time-scale as shown in Figure B7.1.7A. The phase accumulated by a constant velocity isochromat up to a time $t^{\prime}=0$ in the presence of a general phase encoding gradient waveform $G_{y}\left(t^{\prime}\right)$ is given by:

$$
\left.\phi_{v}\left(t^{\prime}\right)\right|_{t^{\prime}=0}=-\left.\gamma v_{y} M_{1}\left(t^{\prime}\right)\right|_{t^{\prime}=0}
$$

where $M_{1}\left(t^{\prime}\right)$ is the first moment of the gradient waveform $G_{y}(t)$ evaluated at time $t=t^{\prime}$ :

$$
M_{1}\left(t^{\prime}\right)=\int_{-\infty}^{t^{\prime}} t G_{y}(t) d t
$$

For the phase encoding gradient waveform in Figure B7.1.7, using $t^{\prime}{ }_{1}=\left(t_{1}-T_{\mathrm{E}}\right)$ to refer

Phase Dependence on Motion B7.1.10 to the time at the end of the gradient in the primed time frame, the first moment $M_{1}\left(t^{\prime}{ }_{1}\right)$ is given by: 


$$
M_{1}\left(t_{1}^{\prime}\right)=\frac{G_{y}}{2}\left(2 \tau_{\mathrm{pe}} t_{1}^{\prime}-\tau_{\mathrm{pe}}^{2}\right)
$$

This implies that the accumulated phase is:

$$
\begin{aligned}
\phi_{v}\left(t_{1}^{\prime}\right) & =-\gamma v_{y} G_{y} \tau_{\mathrm{pe}}\left(t_{1}^{\prime}-\frac{\tau_{\mathrm{pe}}}{2}\right) \\
& =-2 \pi k_{y}\left[v_{y}\left(t_{1}^{\prime}-\frac{\tau_{\mathrm{pe}}}{2}\right)\right] \\
& \equiv-2 \pi k_{y} \Delta y_{v}
\end{aligned}
$$

since $k_{y} \equiv \Varangle G_{y} \tau_{\mathrm{pe}}$. The shift term, $\Delta y_{v}$, is then:

$$
\begin{aligned}
\Delta y_{v} & =v_{y}\left(t_{1}^{\prime}-\frac{\tau_{\mathrm{pe}}}{2}\right) \\
& \equiv v_{y} t_{\mathrm{pe}}^{\prime}
\end{aligned}
$$

where $t^{\prime}{ }_{1}$ is a negative number. The measured $k$-space signal for the flowing spins viewed as a function of $k_{y}$ alone, $s_{v}\left(k_{y}\right)$, is:

$$
\begin{aligned}
s_{v}\left(k_{y}\right) & =s_{s}\left(k_{y}\right) e^{i \phi_{v}\left(t_{1}\right)} \\
& =s_{s}\left(k_{y}\right) e^{-i 2 \pi k_{y} \Delta y_{v}}
\end{aligned}
$$

where $s_{s}\left(k_{y}\right)$ represents the $k$ space data for stationary blood. Hence, the vessel shifts by an amount $\Delta y_{v}$ in the $y$-direction according to the Fourier transform shift theorem. This solution indicates that, effectively, the instant at which phase encoding occurs for constant velocity spins is exactly half-way through the phase encoding gradient.

\section{Ghosting Due to Periodic Motion}

Flow artifacts generally occur because the spins are not located at the same position throughout the encoding process. The most obvious effect is one of blurring. However, another artifact occurs because of the reconstruction method, and that is ghosting. Regardless of the direction of motion, this ghosting appears along the phase encoding direction. There are two sources of ghosting. One is the fact that the phase varies from one phase encoding step to another (see Equation B7.1.25 below) and the other comes from variations in the signal intensity caused by changes in effective magnetization from pulse-to-pulse. The latter can be caused by a variable saturation of spins from pulse-to-pulse, for example.

An interesting flow artifact is found if the effect of motion on the readout data varies from one $T_{\mathrm{R}}$ to the next with a given periodicity. Regardless of the direction of the flow, or translational motion, the artifacts it creates will manifest themselves along the phase encoding direction as ghosts. A discussion of how ghosting would occur for the case of uncompensated flow is presented below. Fortunately, using velocity compensation techniques (UNIT B7.2), these ghosts can be minimized.

\section{Ghosting due to periodic flow}

In practice, flow rates in blood vessels change during the cardiac cycle (of period $T$ ) due to the pulsatile nature of the flow. Consider motion along the read or slice select directions.

Motion Artifacts, Motion Compensation and Magnetic Resonance Angiography

\section{B7.1.11}


For $T_{\mathrm{R}}=T / 2$, all even $k_{y}$ steps will have phase $\phi_{1}$ such that $\phi_{1}=-\gamma \mathrm{G} v_{1} \tau^{2}$ (see Equation $\mathrm{B} 7.1 .11$ ) and all odd $k_{y}$ lines will have phase $\phi_{2}$ such that $\phi_{2}=-\gamma \mathrm{G} v_{2} \tau^{2}$. Here $v_{1}$ is the plug flow velocity along the gradient direction during the first repeat time, during which even lines are acquired, and $v_{2}$ is the plug flow velocity during the second repeat time, during which odd lines are acquired. The gradient $G$ is the amplitude of a simple bipolar pulse which occurs either in the read (Fig. B7.1.1A) or slice select directions. This phase variation leads to aliasing of the vessel.

In general, if $T_{\mathrm{R}}=T / n$, there will be $n$ multiple images (ghosts) across the field-of-view $L_{y}$ in the phase encoding direction, each separated from the other by a distance of $L_{y} / n$ (see Fig. B7.1.2)-i.e.:

$$
\Delta y(p)=p\left(\frac{T_{R}}{T}\right) L_{y}=\frac{p}{n} L_{y}
$$

where $p$ is an integer running from minus infinity to infinity. The reconstructed image will contain information from $\Delta y(p)$ by summing through the whole range of $p$. The reason that there are $n$ ghosts is that the data are sampled at $n$-different locations periodically. (Actually, when $p / n$ is an integer in Equation B7.1.25, there is no phase shift: the original image with a lower magnitude is obtained in those cases.) Fourier transforming this data then leads to aliasing which creates the $n$ objects. From Equation B7.1.25, one can also see that when $p$ is larger than $n / 2$, the object center will be folded (aliased) back within the field-of-view and appear on the left side of the original image. Figure B7.1.2A shows multiple ghosts from the femoral artery. They are closely spaced as indicated by the arrows. They are of low amplitude but still high enough to be bothersome. They are caused by the variations in flow rate from the pulsatility of the arterial flow. This changes the effective periodically changing phase from pulse to pulse causing the ghosting. They are effectively gone in Figure B7.1.2B when flow compensation is used.

The above discussion helps understand the positioning of the ghosts but does not give a prediction of their amplitudes. Fortunately, in this case, where only flow is assumed to be involved, velocity compensation, as discussed in UNIT B7.2, will eliminate the phase differences between different phase encoding lines, and the ghosts will largely, but not completely be eliminated.

Another method to reduce ghosting is to choose a short $T_{\mathrm{R}}$ and average the data over $N_{\text {acq }}$ such that $N_{\text {acq }} T_{\mathrm{R}}$ is roughly $T$ (i.e., $n=N_{\text {acq }}$ ). In this case, only a blur of the image will occur. The spatial extent of this blur is equal to the motion during the acquisition (Fig. B7.1.4A). When the total acquisition is $\sim 15 \mathrm{sec}$ or less, a breath-hold image is possible (Fig. B7.1.4B). In this case, no motion from respiration remains and an excellent image is obtained.

For motion along the phase encoding direction, similar ghosts will occur. These follow the same general characteristics and are in the same direction (the phase encoding) as those caused by motion along $\hat{x}$ (see Figs. B7.1.5A and B7.1.5C).

\section{Examples of ghosting from pulsatile flow}

Even with velocity compensation, ghosting can still occur due to flow. For example, if $\rho(x, y)$ becomes a periodic function of time due to varying inflow (see UNIT B7.3 for a detailed description of inflow effects), then the measured $k$-space signal will vary from one phase encoding line to the next, leading to ghosting (see Fig. B7.1.6). The spacing of the ghosting still obeys Equation B7.1.25.

\section{B7.1.12}


If each given phase encoding line is acquired twice for purposes of averaging, then the ghosts will behave as if the $T_{\mathrm{R}}$ in Equation B7.1.25 were effectively twice the actual value-i.e., $T_{\mathrm{R} \text {,eff }}=2 T_{\mathrm{R}}$. For $N_{\mathrm{acq}}$ acquisitions, $T_{\mathrm{R} \text {,eff }}=N_{\mathrm{acq}} T_{\mathrm{R}}$. If 3-D imaging is performed, $T_{\mathrm{R} \text {,eff }}=N_{\mathrm{z}} T_{\mathrm{R}}$ should be used to compute the ghost positions with $N_{z}$ the number of 3-D partitions. Ghosting from pulsatile (time-varying, but periodic) flow will be dramatically reduced if $T_{\mathrm{R}, \text { eff }}$ equals the period of the flow variation (physiologically, the pulsatile flow period is determined by the cardiac cycle). In other words, if the signal variation is periodic, as $T_{\mathrm{R} \text {,eff }}$ increases, the ghosts get farther apart and eventually vanish for $T_{\mathrm{R}, \mathrm{eff}}=T$ (see Equation B7.1.25).

The effects of motion (spin dephasing and ghosting), as just described, are most dramatic in 2-D slice selective imaging because of the large slice select gradients used for thin slices. An example of the ghosting for a short $T_{\mathrm{R}}$ gradient echo imaging sequence is shown in Figure B7.1.6 for two different $T_{\mathrm{R}}$ values to illustrate both the dependence of the ghost amplitude and position on $T_{\mathrm{R}}$ relative to $T$, the period of the motion. Two comments are made here. The first is that the artifacts which appear in Figure B7.1.6C are worse than the artifacts in the other images in Figure B7.1.6 because Figure B7.1.6C was not velocity compensated. (Velocity compensation is the topic of UNIT B7.2.) The second is that the ghosts in Figure B7.1.6B and Figure B7.1.6D are spaced twice as far apart as those in Figure B7.1.6A and Figure B7.1.6C, because $T_{\mathrm{R}, \text { eff }}$ of the former is twice as large as the latter. Also in this figure, all images show how the ghosts can add coherently (see upper ghosts in the muscle in Fig. B7.1.6B and Fig. B7.1.6D) or cancel muscle signal as in Figure B7.1.6A and Figure B7.1.6C where the black lobes appear.

\section{KEY REFERENCES}

Haacke, E.M., Brown, R.W., Thompson, M.R., and Venkatesan, R. 1999. Magnetic Resonance Imaging: Physical Principles and Sequence Design. John Wiley \& Sons, New York.

This text covers the technical aspects presented here, but in more detail, and also discusses more advanced materials.

Hahn, E.L. 1960. Detection of sea-water motion by nuclear precession. Geophys. Res. 65: 776.

This historical paper introduced phase changes for spins flowing along the direction of the applied magnetic field gradient.

Contributed by Yu-Chung Norman Cheng and E. Mark Haacke

Case Western Reserve University

Cleveland, Ohio

and The MRI Institute for Biomedical Research

St. Louis, Missouri

Motion Artifacts,

Motion

Compensation

and Magnetic

Resonance

Angiography

B7.1.13

Current Protocols in Magnetic Resonance Imaging

Supplement 13 